\title{
Galactic dynamo and helicity losses through fountain flow
}

\author{
A. Shukurov ${ }^{1,3}$, D. Sokoloff $f^{2,3}$, K. Subramanian ${ }^{3}$, and A. Brandenburg ${ }^{4}$ \\ 1 School of Mathematics and Statistics, University of Newcastle, Newcastle upon Tyne, NE1 7RU, UK \\ e-mail: anvar.shukurov@ncl.ac.uk \\ 2 Department of Physics, Moscow University, 119992 Moscow, Russia \\ 3 Inter-University Centre for Astronomy and Astrophysics, Post Bag 4, Ganeshkhind, Pune 411 007, India \\ ${ }^{4}$ Nordita, Blegdamsvej 17, 2100 Copenhagen Ø, Denmark
}

Received 23 December 2005 / Accepted 19 January 2006

\section{ABSTRACT}

Aims. Nonlinear behaviour of galactic dynamos is studied, allowing for magnetic helicity removal by the galactic fountain flow. Methods. A suitable advection speed is estimated, and a one-dimensional mean-field dynamo model with dynamic $\alpha$-effect is explored. Results. It is shown that the galactic fountain flow is efficient in removing magnetic helicity from galactic discs. This alleviates the constraint on the galactic mean-field dynamo resulting from magnetic helicity conservation and thereby allows the mean magnetic field to saturate at a strength comparable to equipartition with the turbulent kinetic energy.

Key words. magnetic fields - turbulence - ISM: magnetic fields - galaxies: ISM

\section{Introduction}

The major controversy in mean-field dynamo theory is related to its nonlinear form relevant when the initial exponential growth of the large-scale magnetic field saturates and the field reaches statistical equilibrium. The core of the problem is the effect of the small-scale (turbulent) magnetic field on the evolution of the large-scale (mean) magnetic field. It has been argued (Vainshtein \& Cattaneo 1992; Cattaneo \& Hughes 1996) that the Lorentz force due to the rapidly growing smallscale magnetic field $\boldsymbol{b}$ can make the large-scale dynamo action inefficient so that it produces only a negligible mean field $\overline{\boldsymbol{B}}$, with $\overline{\boldsymbol{B}}^{2} \simeq R_{\mathrm{m}}^{-1} \overline{\boldsymbol{b}^{2}}$, where $R_{\mathrm{m}}(\gg 1)$ is the magnetic Reynolds number. Here and below, overbars denote averaged quantities. In particular, the $\alpha$-effect (a key ingredient of the mean-field dynamo) is catastrophically quenched well before the largescale magnetic field can be amplified to the strength observed in astrophysical objects, $\bar{B} \simeq b$.

The suppression of the $\alpha$-effect can be a consequence of the conservation of magnetic helicity in a medium of high electric conductivity (see Brandenburg \& Subramanian 2005a for a review). In a closed system, magnetic helicity can only evolve on the Ohmic time scale which is proportional to $R_{\mathrm{m}}$; in galaxies, this time scale by far exceeds the Hubble time. Since the large-scale magnetic field necessarily has non-zero helicity in each hemisphere through the mutual linkage of poloidal and toroidal fields, the dynamo also has to produce small-scale helical magnetic fields with the opposite sign of magnetic helicity.
Unless the small-scale magnetic field can be transported out of the system, it quenches the $\alpha$-effect together with the meanfield dynamo.

Blackman \& Field (2000) first suggested that the losses of the small-scale magnetic helicity through the boundaries of the dynamo region can be essential for mean-field dynamo action. Such a helicity flux can result from the anisotropy of the turbulence combined with large-scale velocity shear (Vishniac \& Cho 2001; Subramanian \& Brandenburg 2004) or the nonuniformity of the $\alpha$-effect (Kleeorin et al. 2000). The effect of the Vishniac-Cho flux has already been confirmed in simulations, allowing the production of significant fields, $\bar{B} \simeq b$ (Brandenburg 2005).

Here we suggest another simple mechanism where the advection of small-scale magnetic fields (together with the associated magnetic helicity) away from the dynamo region allows healthy mean-field dynamo action. This effect naturally arises in spiral galaxies where magnetic field is generated in the multi-phase interstellar medium. The mean magnetic field is apparently produced by the motions of the warm gas (Sect. 4.3 in Beck et al. 1996), which is in a state of (statistical) hydrostatic equilibrium with a scale height of $h \simeq 0.5 \mathrm{kpc}$ (e.g. Korpi et al. 1999). However, some of the gas is heated by supernova explosions producing a hot phase whose isothermal scale height is $3 \mathrm{kpc}$. The hot gas leaves the galactic disc, dragging along the small-scale part of the interstellar magnetic field. Thus, the disc-halo connection in spiral galaxies represents a mechanism of transport of small-scale magnetic fields 
and small-scale magnetic helicity from the dynamo active disc to the galactic halo. As we show here, this helps to alleviate the catastrophic $\alpha$-quenching under realistic parameters of the interstellar medium.

\section{The disc-halo connection in spiral galaxies}

The hot gas produced by supernovae cannot be confined to the galactic disc and therefore it is involved in systematic vertical motions. The gas flows to the halo where it cools and contracts at a height of several kiloparsecs and then falls down to the disc in the form of cold, relatively small clouds, forming what is known as the galactic fountain (Shapiro \& Field 1976; Bregman 1980). The initial vertical velocity of the hot gas is $u_{z}=100-200 \mathrm{~km} \mathrm{~s}^{-1}$ (Norman \& Ikeuchi 1989; Kahn \& Brett 1993; Avillez \& Berry 2001).

The fountain flow drives gas mass flux from the disc, $\dot{M}=$ $2 \pi R^{2} \rho_{\mathrm{h}} f u_{z}$ through both surfaces, where $f$ is the area covering factor of the hot gas, $R \simeq 15 \mathrm{kpc}$ is the radius of the galactic disc with vigorous supernova activity, and $\rho_{\mathrm{h}} \simeq 1.7 \times 10^{-27} \mathrm{~g} \mathrm{~cm}^{-3}$ is the hot gas density. A lower estimate of the area covering factor is given by the volume filling factor of the hot gas at the disc midplane, $f=0.2-0.3$ (e.g. Korpi et al. 1999), because the scale height of the galactic disc is comparable to the size of the hot cavities. This yields $\dot{M} \simeq 1.5 M_{\odot} \mathrm{yr}^{-1}$ (cf. Norman \& Ikeuchi 1989 who obtain $\dot{M}=0.3-3 M_{\odot} \mathrm{yr}^{-1}$ ).

The dynamo model discussed below refers to quantities averaged over scales exceeding the size of the hot cavities, and so it treats the multi-phase interstellar medium in an averaged manner. Then it is appropriate to introduce an effective fountain speed as the one that drives the same mass flux as above by advecting the diffuse interstellar gas at its mean density, $\rho=1.7 \times 10^{-25} \mathrm{~g} \mathrm{~cm}^{-3}$ (i.e. number density of $0.1 \mathrm{~cm}^{-3}$ )

$U_{0}=f u_{z} \frac{\rho_{\mathrm{h}}}{\rho} \simeq 1-2 \mathrm{~km} \mathrm{~s}^{-1}$,

and less for larger $\rho$ (say, $0.3-0.7 \mathrm{~km} \mathrm{~s}^{-1}$ for the gas number density of $\left.0.3 \mathrm{~cm}^{-3}\right)$. Given the uncertainty of the magnitude of $f$ (e.g., $f \simeq 0.1$ in the model of Norman \& Ikeuchi 1989), the range $U_{0}=0.2-2 \mathrm{~km} \mathrm{~s}^{-1}$ seems to be plausible.

The hot gas that leaves the galactic disc carries magnetic fields of scales smaller than the size of the hot cavities $(0.1-1 \mathrm{kpc})$; these are mostly turbulent magnetic fields (of scales $\lesssim 100 \mathrm{pc}$ ). The time scale of the removal of the smallscale magnetic fields is of order $h / U_{0} \simeq 5 \times 10^{8} \mathrm{yr}$ (with $h \simeq 0.5 \mathrm{kpc}$ the scale height of the warm gas layer which hosts the mean-field dynamo), which is comparable to the turbulent diffusion time of the mean field, $h^{2} / \eta_{\mathrm{t}} \simeq 8 \times 10^{8} \mathrm{yr}$, with $\eta_{\mathrm{t}}=10^{26} \mathrm{~cm}^{2} \mathrm{~s}^{-1}$ the turbulent magnetic diffusivity. Hence, the leakage of the small-scale magnetic helicity produced by the galactic fountain can significantly affect the mean-field dynamo.

\section{Magnetic helicity balance with fountain flow}

In the following we use, wherever appropriate, lower case characters to denote random, small scale quantities defined as the departure from the corresponding mean, e.g. $\boldsymbol{a}=\boldsymbol{A}-\overline{\boldsymbol{A}}$,
$\boldsymbol{j}=\boldsymbol{J}-\overline{\boldsymbol{J}}$ and $\boldsymbol{u}=\boldsymbol{U}-\overline{\boldsymbol{U}}$ for the vector potential, current density and velocity, respectively. A gauge-invariant magnetic helicity density $\chi$ can be defined for $\boldsymbol{b}$ as the density of correlated links of $\boldsymbol{b}$ using Gauss's linkage formula (Subramanian $\&$ Brandenburg 2005). Such a definition can be introduced for random magnetic fields if their correlation length is finite and much smaller than the system size. The helicity density $\overline{\boldsymbol{a} \cdot \boldsymbol{b}}$ defined with the Coulomb gauge, $\boldsymbol{\nabla} \cdot \boldsymbol{a}=0$, is only approximately the same as $\chi$. In the presence of a helicity flux $\boldsymbol{F}$, the magnetic helicity density for the small-scale field evolves as (Subramanian \& Brandenburg 2005)

$\frac{\partial \chi}{\partial t}+\boldsymbol{\nabla} \cdot \boldsymbol{F}=-2 \mathcal{E} \cdot \overline{\boldsymbol{B}}-2 \eta \overline{\boldsymbol{j} \cdot \boldsymbol{b}}$

where $\mathcal{E}=\overline{\boldsymbol{u} \times \boldsymbol{b}}$ is the turbulent emf and $\eta$ is the Ohmic magnetic diffusivity. (We use units where $\boldsymbol{J}=\nabla \times \boldsymbol{B}$.) In the steady state, Eq. (2) yields $\mathcal{E} \cdot \overline{\boldsymbol{B}}=-\frac{1}{2} \boldsymbol{\nabla} \cdot \boldsymbol{F}-\eta \overline{\boldsymbol{j} \cdot \boldsymbol{b}}$. Without a helicity flux, $\boldsymbol{E} \cdot \overline{\boldsymbol{B}}=-\eta \overline{\boldsymbol{j} \cdot \boldsymbol{b}}$ follows, which vanishes as $\eta \rightarrow 0$ for any reasonable spectrum of current helicity $\overline{\boldsymbol{j} \cdot \boldsymbol{b}}$ so that $\overline{\boldsymbol{j} \cdot \boldsymbol{b}} \propto \eta^{-\kappa}$ with $\kappa<1$. In the presence of helicity fluxes, however, $\mathcal{E} \cdot \overline{\boldsymbol{B}}$ needs not be catastrophically quenched as $\eta \rightarrow 0$.

We study the effects of the simplest contribution to the flux,

$\boldsymbol{F}=\chi \overline{\boldsymbol{U}}$

which arises from the net effect of advection by upward and downward flows (cf. Subramanian \& Brandenburg 2005) dominated by the upward flow in the case of galactic fountains. The overall effect of the resulting helicity advection from the disc of the galaxy is not immediately obvious since the flow can also remove the mean magnetic field from the dynamo-active region. To reveal the net effect of the fountain flow on the dynamo we solve the helicity equation simultaneously with the mean-field dynamo equation,

$\frac{\partial \overline{\boldsymbol{B}}}{\partial t}=\boldsymbol{\nabla} \times(\overline{\boldsymbol{U}} \times \overline{\boldsymbol{B}}+\mathcal{E}-\eta \overline{\boldsymbol{J}})$

We adopt $\mathcal{E}=\alpha \overline{\boldsymbol{B}}-\eta_{\mathrm{t}} \overline{\boldsymbol{J}}$ (assuming isotropic turbulence) and take $\alpha=\alpha_{\mathrm{K}}+\alpha_{\mathrm{m}}$ (following Pouquet et al. 1976), where $\alpha_{\mathrm{K}}$ represents the kinetic $\alpha$-effect, $\alpha_{\mathrm{m}}=\frac{1}{3} \rho^{-1} \overline{\boldsymbol{\tau} \cdot \boldsymbol{b}}$ is the magnetic contribution to the $\alpha$-effect, and $\eta_{\mathrm{t}}=\frac{1}{3} \overline{\tau \boldsymbol{u}^{2}}$.

The dynamics of $\alpha_{\mathrm{m}}$ is controlled by Eq. (2). We argue that the main contribution to $\alpha_{\mathrm{m}}$ comes from the integral scale of the turbulence $l_{0}=2 \pi / k_{0}$. For Kolmogorov turbulence, we have the following spectral scalings: $\boldsymbol{j}_{k} \cdot \boldsymbol{b}_{k} \propto k b_{k}^{2} \propto k^{1 / 3}$ and $\tau_{k} \propto k^{-2 / 3}$, so that $\alpha_{\mathrm{m}} \propto k^{-1 / 3}$. Moreover, numerical results of Brandenburg \& Subramanian (2005b) indicate that even $\overline{\boldsymbol{j} \cdot \boldsymbol{b}}$ is dominated by the larger scales. This justifies the estimate

$\alpha_{\mathrm{m}} \simeq \frac{1}{3} \tau k_{0}^{2} \frac{\chi}{\rho}$.

With $B_{\mathrm{eq}}^{2} \equiv \rho \overline{u^{2}}$ and $R_{\mathrm{m}}=\eta_{\mathrm{t}} / \eta$, we then rewrite Eq. (2) as (cf. Blackman \& Brandenburg 2002; Subramanian 2002),

$\frac{\partial \alpha_{\mathrm{m}}}{\partial t}=-2 \eta_{\mathrm{t}} k_{0}^{2}\left(\frac{\mathcal{E} \cdot \overline{\boldsymbol{B}}}{B_{\mathrm{eq}}^{2}}+\frac{\alpha_{\mathrm{m}}}{R_{\mathrm{m}}}\right)-\boldsymbol{\nabla} \cdot\left(\alpha_{\mathrm{m}} \overline{\boldsymbol{U}}\right)$. 
Since galactic discs are thin, it suffices to consider a onedimensional model, retaining only the $z$-derivatives of the variables (Ruzmaikin et al. 1988). In terms of cylindrical coordinates $(r, \phi, z)$, we adopt a mean flow consisting of rotation (shear) and vertical advection, with $\overline{\boldsymbol{U}}=\left(0, \bar{U}_{\phi}, \bar{U}_{z}\right)$, where $\bar{U}_{z}=U_{0} z / h$ within the disc. We use $\alpha_{\mathrm{K}}=\alpha_{0} z / h$. The meanfield dynamo equation, in cylindrical coordinates, becomes

$$
\begin{aligned}
& \frac{\partial \bar{B}_{r}}{\partial t}=-\frac{\partial}{\partial z}\left(\bar{U}_{z} \bar{B}_{r}+\mathcal{E}_{\phi}\right)+\eta \frac{\partial^{2} \bar{B}_{r}}{\partial z^{2}}, \\
& \frac{\partial \bar{B}_{\phi}}{\partial t}=-\frac{\partial}{\partial z}\left(\bar{U}_{z} \bar{B}_{\phi}-\mathcal{E}_{r}\right)+\eta \frac{\partial^{2} \bar{B}_{\phi}}{\partial z^{2}}+q \Omega_{0} \bar{B}_{r},
\end{aligned}
$$

where $q \approx-1$ for a flat rotation curve, $\Omega \propto r^{-1}$, and $\Omega_{0}$ is the local rotation rate. We solve Eqs. (4)-(6) with the boundary conditions

$$
\bar{B}_{r}=\bar{B}_{\phi}=0 \quad \text { at } z= \pm h .
$$

We adopt $\alpha_{\mathrm{m}}=0$ at $t=0$, and a symmetry condition $\alpha_{\mathrm{m}}=0$ at $z=0$ follows automatically from the symmetry of $\overline{\boldsymbol{B}}$; the two conditions result in a unique solution of Eq. (4). (There is no need to be specify separately $\alpha_{\mathrm{m}}$ at $z= \pm h$.) We introduce dimensionless numbers

$C_{U}=\frac{U_{0}}{\eta_{\mathrm{t}} k_{1}}, \quad C_{\Omega}=\frac{\Omega_{0}}{\eta_{\mathrm{t}} k_{1}^{2}}, \quad C_{\alpha}=\frac{\alpha_{0}}{\eta_{\mathrm{t}} k_{1}}$,

where $k_{1}=\pi / h$. For the fiducial values $h=500 \mathrm{pc}, U_{0}=$ $0.2-2 \mathrm{~km} \mathrm{~s}^{-1}, \Omega_{0}=25 \mathrm{~km} \mathrm{~s}^{-1} \mathrm{kpc}^{-1}, \alpha_{0}=0.5 \mathrm{~km} \mathrm{~s}^{-1}$ and $\eta_{\mathrm{t}}=$ $10^{26} \mathrm{~cm}^{2} \mathrm{~s}^{-1}$, we have $C_{U}=0.1-1, C_{\Omega} \approx-2$ and $C_{\alpha} \approx 0.8$. We use $k_{0} / k_{1}=h / l_{0}=5$ as appropriate for the galactic disc, and present our results in the units of

$B_{\mathrm{eq}} \approx 5 \mu \mathrm{G}$ and $\left(\eta_{\mathrm{t}} k_{1}^{2}\right)^{-1} \approx 8 \times 10^{7} \mathrm{yr}$

for magnetic field and time, respectively (where gas number density of $1 \mathrm{~cm}^{-3}$ and the turbulent velocity of $10 \mathrm{~km} \mathrm{~s}^{-1}$ has been used in $B_{\text {eq }}$ ).

\section{Results}

We compare in Fig. 1 the evolution, with and without the advective flux, of $\left\langle\overline{\boldsymbol{B}}^{2}\right\rangle / B_{\text {eq }}^{2}$, where $\left\langle\overline{\boldsymbol{B}}^{2}\right\rangle$ is the mean square of the large-scale magnetic field over the range $|z|<h$. The solutions are non-oscillatory and have quadrupolar parity; they grow for $C_{\alpha}>0.2$ if $C_{\Omega}=-2$ and $C_{U}=0$. For the fiducial parameters, the $e$-folding time of the dynamo is about $3 \times 10^{8} \mathrm{yr}$ for $C_{U} \lesssim 0.3$. In the absence of an advective flux, the initial growth of magnetic field is catastrophically quenched and the largescale magnetic field decreases at about the same rate as it grew. The initial growth occurs while the current helicity builds up to cancel the kinetic $\alpha$-effect. However, even a modest advective flux $\left(C_{U}=0.1\right)$ compensates the catastrophic quenching of the dynamo and the mean field energy density stays steady at about $10^{-2} B_{\text {eq }}^{2}$, which corresponds to the field strength of about $10 \%$ of the equipartition value.

We illustrate in the inset of Fig. 1 the effect of varying the strength of the advection velocity on the dynamo. The steadystate strength of the mean field grows with $U_{0}$ as the advection

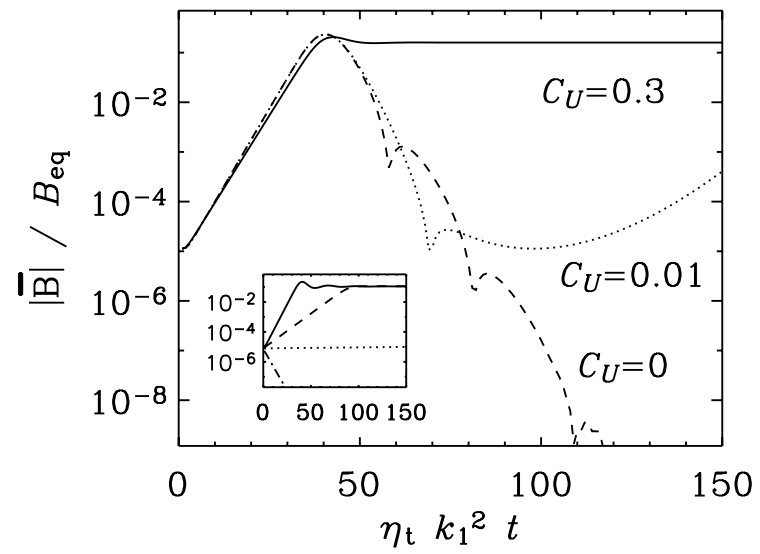

Fig. 1. Evolution of the field strength at $z=0$ obtained by solving Eqs. (4)-(7) with vertical advection (solid line, $C_{U}=0.3$ ) and without it (dashed line, $C_{U}=0$ ), for $C_{\Omega}=-2, C_{\alpha}=1$ and $R_{\mathrm{m}}=10^{5}$. The dynamo is neutrally stable at $C_{\alpha}=0.26$ for $\mathrm{C}_{U}=0.3$ and $C_{\Omega}=2$. The dotted curve, obtained for $C_{U} \ll 1$, shows that even weak advection can affect the long-term evolution of magnetic field. For $\mathrm{C}_{U}=0$, nonlinear effects make the $\alpha$ profile flatter at small $|z|$; this causes an oscillatory decay of the field. The inset shows similar results for $\mathrm{C}_{U}=$ 0.1 (solid), 1.5 (dashed), 2 (dotted) and 3 (dash-dotted).

strength increases to about $C_{U}=0.3$. For stronger advection, $C_{U} \simeq 0.5$, the mean field initially grows slower but still attains a steady state strength slightly exceeding $0.1 B_{\text {eq }}$. Stronger advection, $C_{U}>1$, affects the dynamo adversely since the mean field is removed too rapidly from the dynamo active region. A good compromise between rapid growth and large saturation field strength is reached for $C_{U} \approx 0.1$, which is close to the values expected for spiral galaxies.

Modest advection does not noticeably affect the spatial distribution of magnetic field. The profiles of $\bar{B}_{\phi}$ and $\bar{B}_{r}$ shown in Fig. 2 for the steady state do not differ much from solutions of the kinematic dynamo equations (cf. Ruzmaikin et al. 1988). The corresponding profiles of $\alpha$ and $\alpha_{\mathrm{m}}$ shown in the lower panel, indicate that the suppression of $\alpha$ in stronger near the disc midplane (at small $z$ ), where magnetic field is stronger.

The steady-state strength of $\overline{\boldsymbol{B}}$, can be estimated from magnetic helicity conservation. Averaging Eq. (4), with $\partial \alpha_{\mathrm{m}} / \partial t=$ 0 , over $z$ on $0<z<h$ (with the mean denoted with angular brackets) yields

$\frac{\langle\boldsymbol{E} \cdot \overline{\boldsymbol{B}}\rangle}{B_{\mathrm{eq}}^{2}}+\frac{\left\langle\alpha_{\mathrm{m}}\right\rangle}{R_{\mathrm{m}}}+\frac{\alpha_{\mathrm{m}}(h) \bar{U}_{z}(h)}{2 \eta_{\mathrm{t}} k_{0}^{2} h}=0$,

since $U_{z}(0)=0$. For the adopted boundary conditions (7) and $\left|\bar{B}_{z}\right| \ll\left|\bar{B}_{r}\right|,\left|\bar{B}_{\phi}\right|$ within the disc, it is meaningful to calculate magnetic helicity within the disc only. Advection does not affect helicity conservation, which then implies $\langle\overline{\boldsymbol{J}} \cdot \overline{\boldsymbol{B}}\rangle \simeq$ $k_{1}^{2}\langle\overline{\boldsymbol{A}} \cdot \overline{\boldsymbol{B}}\rangle=-k_{1}^{2} \chi$. Given that $\mathcal{E} \cdot \overline{\boldsymbol{B}} / B_{\mathrm{eq}}^{2} \simeq\left(\alpha_{\mathrm{K}}+\alpha_{\mathrm{m}}\right)\left(\bar{B} / B_{\mathrm{eq}}\right)^{2}+$ $\alpha_{\mathrm{m}}\left(k_{1} / k_{0}\right)^{2}$, and assuming that $\alpha_{\mathrm{K}}+\alpha_{\mathrm{m}}=\alpha_{\mathrm{c}}$, where $\alpha_{\mathrm{c}}$ corresponds to a marginally stable dynamo, Eq. (9) yields the following estimate of magnetic field strength in the steady state:

$\bar{B} \simeq B_{\text {eq }} \frac{k_{1}}{k_{0}}\left(\frac{D}{D_{\mathrm{c}}}-1\right)^{1 / 2} C^{1 / 2}$, 

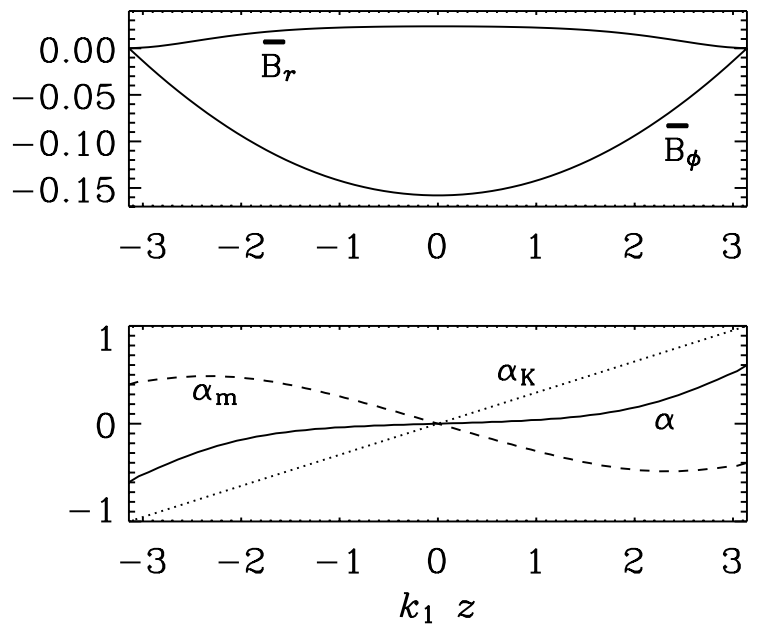

Fig. 2. Plots of $\bar{B}_{\phi}$ and $\bar{B}_{r}$ (upper panel) and $\alpha$ (solid), $\alpha_{\mathrm{m}}$ (dashed) and $\alpha_{\mathrm{K}}$ (dotted) (lower panel) versus $z$ in the steady state for $C_{U}=0.3$, $C_{\Omega}=-2, C_{\alpha}=1, R_{\mathrm{m}}=10^{5}$, and $k_{1}=\pi / h$.

where $D=C_{\alpha} C_{\Omega}$ is the dynamo number and $D_{\mathrm{c}}$ is its marginal value, and $C=1+C_{U} /(2 \pi)+k_{0}^{2} /\left(R_{\mathrm{m}} k_{1}^{2}\right)=O(1)$. With $k_{1} / k_{0} \simeq 5$ and $D \simeq D_{\text {c }}$, this implies $\bar{B} \simeq 0.2 B_{\text {eq }}$ in rough agreement with the numerical solution. We note that Eq. (10) does not apply for $|D| \gg\left|D_{\mathrm{c}}\right|$ because our arguments are only valid for weakly nonlinear regimes.

\section{Discussion}

The vertical advection of magnetic helicity by galactic fountain flow resolves straightforwardly the controversy of nonlinear mean-field galactic dynamos. For $C_{U}=0$, the mean magnetic field does initially reach a level consistent with Eq. (10), but then rapidly decays to negligible values (see Brandenburg \& Subramanian 2005c). The essential role of the advection is to provide the system with an opportunity to reach and then maintain the steady state (10) within the galactic lifetime.

These conclusions follow from Fig. 1, where moderate advection drastically changes the mean field levels achievable at $t \lesssim 10^{10} \mathrm{yr}$ and prevents catastrophic quenching of the dynamo. Excessive advection, however, hinders the dynamo as it removes the mean field from the dynamo active region.

The steady-state strength of the mean magnetic field obtained in our model is of order $(0.1-0.2) B_{\text {eq }} \simeq 0.5-1 \mu \mathrm{G}$, which is a factor of several weaker than what is observed; we made no attempt here to refine the model. What is important, the mechanism suggested here resolves the problem of catastrophic quenching of the dynamo.

The applicability of the vacuum boundary conditions (7) to a system with gas outflow from the disc can be questionable. Analysis of dynamo models with outflow (Bardou et al. 2001) suggests that reasonable changes to the boundary conditions do not affect the dynamo too strongly, but this aspect of our model should be further explored.

We have neglected the intrinsic difference of the behaviours of the mean and turbulent magnetic fields near the disc surface. Since the horizontal size of the hot cavities is larger than the scale of the turbulent magnetic field but smaller than the scale of the mean magnetic field, the Lorentz force can resist the advection of the mean field more efficiently than that of the turbulent field. Furthermore, large-scale magnetic field loops drawn out by the fountain flow can be detached from the parent magnetic lines by reconnection, so that the flow will carry mostly small-scale fields. Therefore, a more plausible (albeit less conservative) model would include advection of the small-scale (but not the large-scale) magnetic field. In such a model, the effect discussed here can be even better pronounced.

Brandenburg et al. (1995) argue that the fountain flow can transport the large-scale magnetic field into the halo by topological pumping if the hot gas forms a percolating cluster in the disc and if the turbulent magnetic Reynolds number $C_{U}$ in the fountain flow exceeds $\simeq 20$; our estimate given below is $C_{U} \lesssim 1$, and we expect that the small-scale magnetic fields are removed from the disc more efficiently than the mean field.

The idea that advection of small-scale magnetic fields can help the galactic dynamo may be more robust than our particular model of dynamo quenching that involves the magnetic $\alpha$-effect. For example, if the dynamo coefficients are quenched due to the suppression of Lagrangian chaos by the small-scale magnetic fields (Kim 1999), their advection out of the galaxy will still allow the dynamo to operate efficiently.

Acknowledgements. We are grateful to David Moss for helpful comments. K.S. acknowledges the hospitality of NORDITA. This work was supported by the Royal Society, RFBR grant 04-02-16094 and INTAS grant 2021.

\section{References}

de Avillez, M. A., \& Berry, D. L. 2001, MNRAS, 328, 708

Bardou, A., von Rekowski, B., Dobler, W., et al. 2001, A\&A, 370, 635

Beck, R., Brandenburg, A., Moss, D., et al. 1996, ARA\&A, 34, 155

Blackman, E. G., \& Field, G. B. 2000, ApJ, 534, 984

Blackman, E. G., \& Brandenburg, A. 2002, ApJ, 579, 359

Brandenburg, A. 2001, ApJ, 550, 824

Brandenburg, A. 2005, ApJ, 625, 539

Brandenburg, A., \& Subramanian, K. 2005a, PhR, 417, 1

Brandenburg, A., \& Subramanian, K. 2005b, A\&A, 439, 835

Brandenburg, A., \& Subramanian, K. 2005c, AN, 326, 400

Brandenburg, A., Moss, D., \& Shukurov, A. 1995, MNRAS, 276, 651

Bregman, J. N. 1980, ApJ, 236, 577

Cattaneo, F., \& Hughes, D. W. 1996, Phys. Rev. E, 54, 4532

Field, G. B., \& Blackman, E. G. 2002, ApJ, 572, 685

Kahn, F. D., \& Brett, L. 1993, MNRAS, 263, 37

Kim, E.-J. 1999, Phys. Lett. A, 259, 232

Kleeorin, N., Moss, D., Rogachevskii, I., et al. 2000, A\&A, 361, L5

Korpi, M. J., Brandenburg, A., Shukurov, A., et al. 1999, ApJ, 514, L99

Norman, C. A., \& Ikeuchi, S. 1989, ApJ, 345, 372

Pouquet, A., Frisch, U., \& Léorat, J. 1976, JFM, 77, 321

Ruzmaikin, A. A., Shukurov, A. M., \& Sokoloff, D. D. 1988, Magnetic Fields of Galaxies (Dordrecht: Kluwer)

Shapiro, P. R., \& Field, G. B. 1976, ApJ, 205, 762

Subramanian, K. 2002, Bull. Astr. Soc. India, 30, 715

Subramanian, K., \& Brandenburg, A. 2004, PRL, 93, 205001

Subramanian, K., \& Brandenburg, A. 2005

[arXiv: astro-ph/0509392]

Vainshtein, S. I., \& Cattaneo, F. 1992, ApJ, 393, 165

Vishniac, E. T., \& Cho, J. 2001, ApJ, 550, 752 